\title{
Smart Runways-Use of Residual Vibration Energy from Air Wake to Produce Electricity
}

\author{
Mohit Agarwal and Jafar Ali
}

\begin{abstract}
The importance of aviation industry in modern era is long familiar. The industrial sector has been suffering energy crises from decades and consequently paves way for the need of energy regeneration and utility. Runways are heart of an airport and currently runways are used as pathways for airplane takeoff and landing. The proposed idea emphasizes use of residual mechanical vibrational energy from aircraft during takeoff and landing. Air Wakes are huge source of vibrational energy and these vibrations are harnessed using energy regenerative engineering technology. The lifting aircraft produces turbulent air wakes, which strike light weight swinging elliptical dish plates along sides of the runway. These are made to vibrate continuously and impact the piezoelectric media which consequently produces electric potential. Varieties of efficient piezoelectric harnessers are being developed using different vibration analysis and many have embarked upon the idea. But this paper is unparallel to those in many ways.
\end{abstract}

Index Terms-Air wakes, piezoelectric materials, Van de Graaff principle.

\section{INTRODUCTION}

The basic concept is to incident the Air Wakes on an elliptical dish plates installed, which deforms the planar grid of the dish structure (stretched grid transformation). The lower portion of rear face of the dish has cylindrical fins having the conical nose cone shape. This are made to hit the piezoelectric material housed at a certain height. The major concern in the mechanism is to ensure the longer period of the sustenance of the impact on the piezoelectric material, this is achieved by placing the dish on a roller joint set up giving the rotary motion and the torsional spring set up on the arm to make the whole dish oscillate continuously which results in the longer impact period and hence net electric potential developed is amplified. First we will discuss what are air wakes and their generation, followed by the piezoelectric materials and Van de Graaff principle. Moving on we shall present the assembly of the components and how they are integrated with the electrical circuits and meshed with each other. Then we shall proceed with the discussion on the grid transformations that takes place and stability analysis of the structure. The math and the complete concept of operations have been explained.

\section{AIR WAKES}

Wake vortex, the horizontal turbulence generated behind

Manuscript received January 26, 2013; revised April 3, 2013.

Mohit Agarwal and Jafar Ali are with SRM University, Kattankulathur,Chennai, India (e-mail: mohitagarwa1937@gmail.com). aircraft, limits airport capacity by making it necessary to space arriving and departing aircraft to keep them from encountering another aircraft's wake. All aircraft produce wake turbulence more correctly wing trip vortices. They are produced when an aerofoil produces lift anytime, by the creation of the pressure differential over the wing surfaces. The tendency of the air to move to the area of the lower pressure causes it to move outwards under the wing towards the wing tip and curl up and over the surface of the wing, leading to the vortex creation (Fig. 1) [1].

\section{Piezoelectric Materials:}

There are certain non conducting materials, such as quartz crystals and ceramics, when subjected to mechanical stress (such as pressure or vibration) develop electric charges, or the generation of vibrations in such materials when they are subjected to an electric field. Piezoelectric materials when exposed to a fairly constant electric field tend to vibrate at a precise frequency with very little variation, making them useful as time-keeping devices wristwatches and also find its application in computers [2], [3].

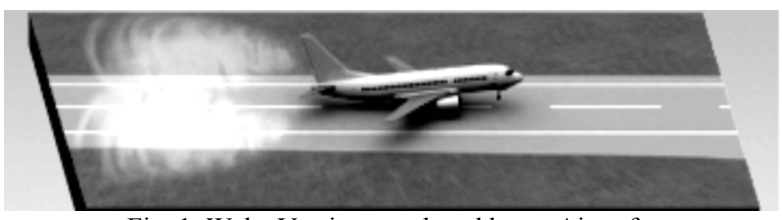

Fig. 1. Wake Vortices produced by an Aircraft

\section{VAN DE GRAAFF PRINCIPLE:}

An electrostatic generator which uses a moving belt to accumulate very high voltages on a hollow metal globe on the top of the stand is called a Van de Graaff generator. The potential difference achieved in modern Van de Graaff generators can reach 5 megavolts. The Van de Graaff generator can be thought of as a constant-current source connected in parallel with a capacitor and very large electrical resistance, so it can produce visible electrical discharge to a nearby grounding surface which can potentially cause a "spark" depending on the voltage. See Fig. 2 [4].

\section{ASSEMBLY}

\section{A. Receptor}

As shown in the Fig. 3. The setup consists of a concave (inward projected) ellipsoidal dish which is initially assumed to be made up of aluminum. The plate comes under continuous influence of various drag forces due to wakes and 
vibrational energy produced by the aircrafts. The elliptical dish is placed on the rotary joint to impart continual swing once actuated for a longer duration. A series of such dishes run along the sideways of the runway. The dish structure is supported by two rods under which springs (assume medium- low stiffness) are mounted rigid on a concrete base.

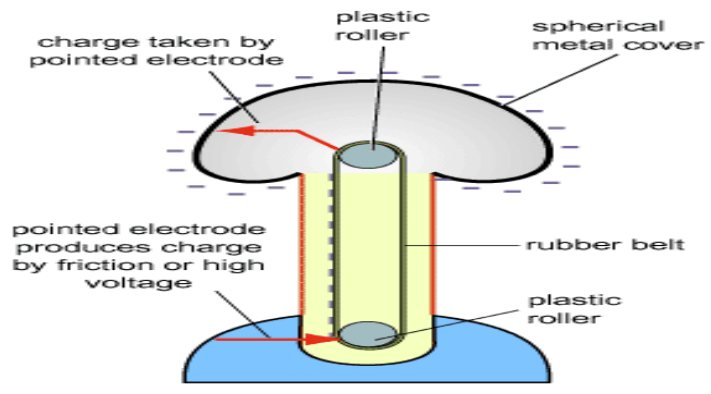

Fig. 2. Vande Graaff Generator

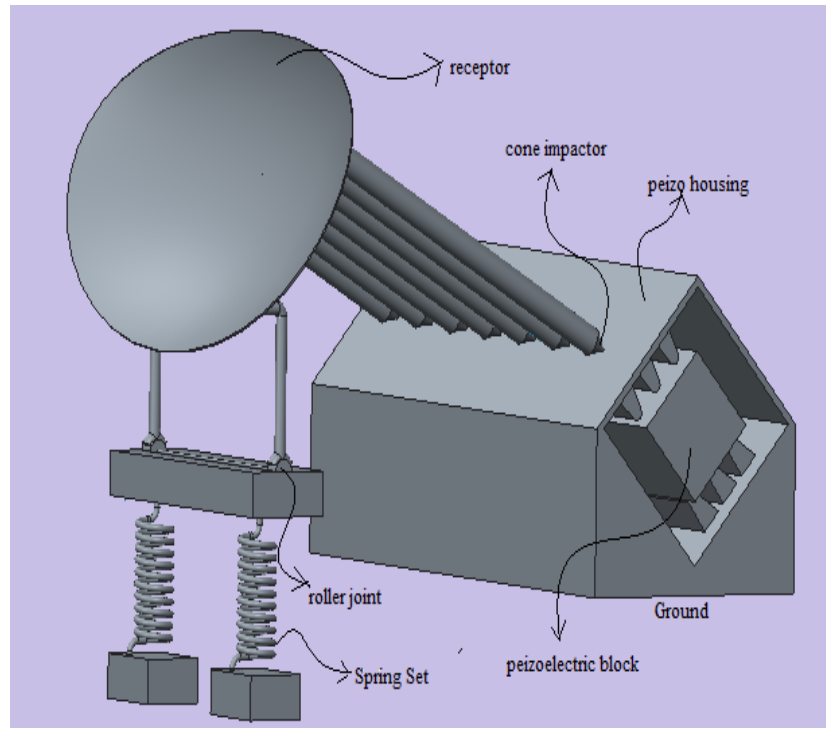

Fig. 3. Structural Design

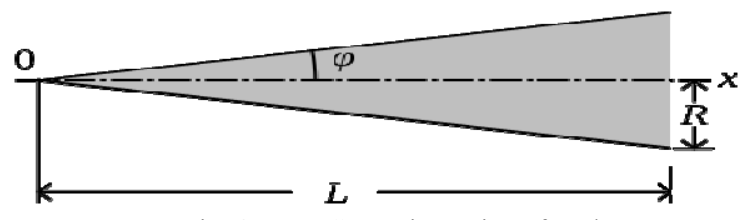

Fig. 4. Nose Cone Tip Design of Rod

\section{B. Transmission}

The wakes received along the runways is at low speed, making it necessary to design a set up to amplify the pressure, thus cylindrical fins with the conical nose tips as shown in the assembly diagram. The equations are listed below in support of the argument. The total drag forces on the dish are thus represented as a function of nodes along the fins and the maximum pressure being produced at the conical end (Fig. 4). This ensures large mechanical pressure being applied at the piezoelectric setup.

\section{Peizoelectric Housinng}

The piezoelectric housing consists of a cubed shaped piezoelectric material placed in a cubical chamber placed along the radial plane of receptor dish. The inner surface of the metal casing again has conical spokes which are designed and aligned such that they are just in contact with the piezoelectric surface. The housing is fixed on a concrete base.
The other two faces of piezoelectric material are connected through a feedback circuit, thus completing the process. Fig. 5 shows the conventional piezoelectric circuit block diagram.

\section{Delivery Circuit (Voltage Doubler):}

The Voltage Doubler is significant improvement over the Villard circuit for a small cost in additional components. The Villard circuit uses only one diode and capacitor and has poor ripple characteristics. The ripple in a doubler is negligible, nominally zero under open-circuit load conditions, but when current is being drawn depends on the resistance of load and the value of the capacitors used. The circuit works by following a Villard cell stage with what is in essence a peak or envelope detector stage. The peak detector cell has the effect of removing most of the ripple while preserving the peak voltage in the output [5].

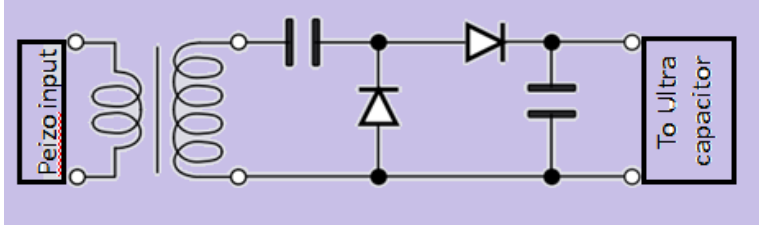

Fig. 5. Sample Delivery Circuit

\section{EQUATIONS AND TRANFORMATIONS}

The elliptical dish is under the influence of the skin and induced drag forces, the forces acting on the set up are: For motions perpendicular to the plane of the disk as shown in Fig. 6 the equation (1) is used

$$
F_{D}=16 \mu a U
$$

For motions along the plane of the disk equation 2

$$
F_{D}=32 / 3 \mu a U
$$

Elliptical grid generation: The elliptical thin dish is made of light weight materials (for eg: $\mathrm{Al}$ ) and the drag induced can damage the grid of the dish. The stretched grid transformation was performed to validate it as a fully safe set up. A simple analytical transformation which can accomplish the grid stretching is shown in the equation (3) and (4).

$$
\begin{gathered}
x=\xi \\
\eta=y / y_{s} \quad \text { where } y_{s}=f(x)
\end{gathered}
$$

The elliptical grid has the vertical grid lines separated equally but the horizontal lines are to be fitted and transformed. Thus, in the control plane of the dish the $x$ coordinates are same but the y coordinates have been represented as function of $(x)$. a simple example can be $y=y_{d}=y_{s}\left(x_{d}\right)$. When this is substituted into Eq 5, we have

$$
\eta_{\mathrm{d}}=y_{d} / y_{s}=y_{s}\left(x_{d}\right) / y_{s}\left(x_{d}\right)=1
$$

Thus in the computational plane, point $\mathrm{d}$ is located along $\eta=\eta_{\mathrm{d}}=1$. When we observe another point $\mathrm{c}$, the ordinates differ in the physical plane (Fig. 7) but in the computational plane(see Fig 8) they all have the same value, $\eta_{\mathrm{c}}=\eta_{\mathrm{d}}=1$ as shown in Fig . 7 and Fig. 8 [6], [7]. 


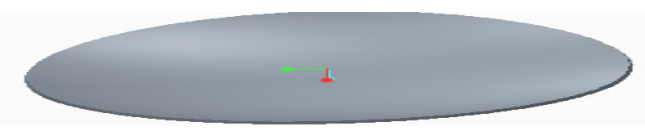

Fig. 6. Elliptical Dish

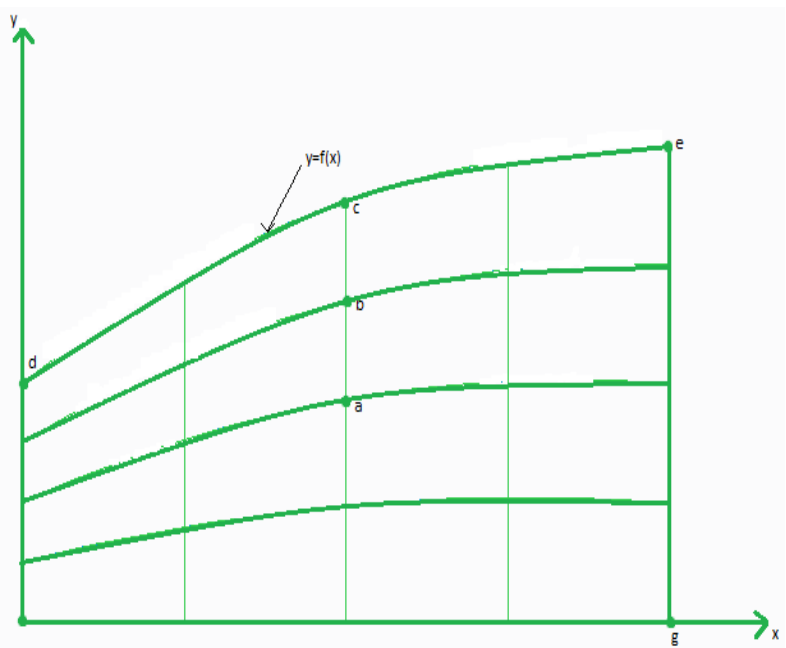

Fig. 7. Physical Elliptical Grid Plane

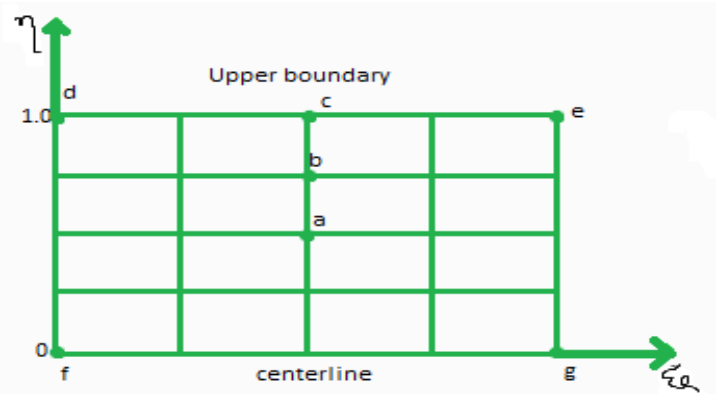

Fig. 8. Control Plane

\section{STRUCTURAL ANALYSIS RESUltS}

The design is subjected to extremely large pressure and endurance of the set up is a major concern. The analysis on the assembly model is carried out in ANSYS 14 workbench. The two simulations carried out are stress and deformation analysis.

Stress Analysis: For stress (von-Mises stress) was found to be maximum at the center of the dish and was found to be $1.1512 \mathrm{Mpa}$. Also the stresses varied between 1.0 to $0.12 \mathrm{Mpa}$ over the dish structure (See Fig. 9). The roller joint of the model was given a zero degree freedom constraint and the dish was given a motion freedom as rotational Y.

The design analysis also proved that the structure can withstand surface loads much higher than expected. Also, the actual force acting on the plate is the resultant of the drag forces acting parallel and perpendicular to the plate. The largest pressure is created at the tip of the nose cone and thus imparting maximum impact, as expected. The following shows the real time result of the stress analysis.

Deformation analysis: The deformation of the ellipsoidal dish was found to be maximum over its top center edge, from where the air wakes would be following the circulatory flow. The deformation of the dish was found to be $0.07078 \mathrm{~mm}$ (See Fig. 10). The analysis was done keeping in mind that the dish is made up of an Aluminum alloy and similar properties of the alloy was fed for the resulting solution and the results of the simulation validated the need of extremely large pressure (much higher than the actual loads) to cause a minor deflection. The real time simulation results follow [8].

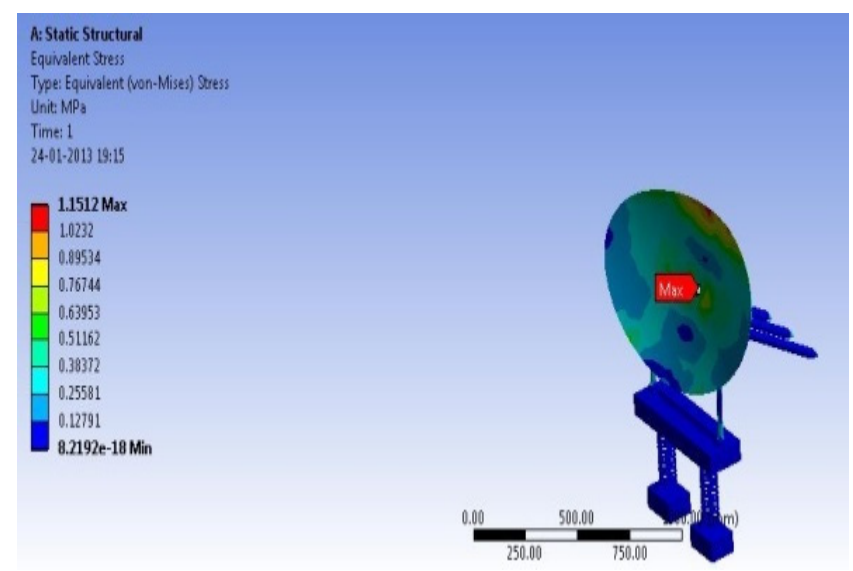

Fig. 9. Stress Analysis

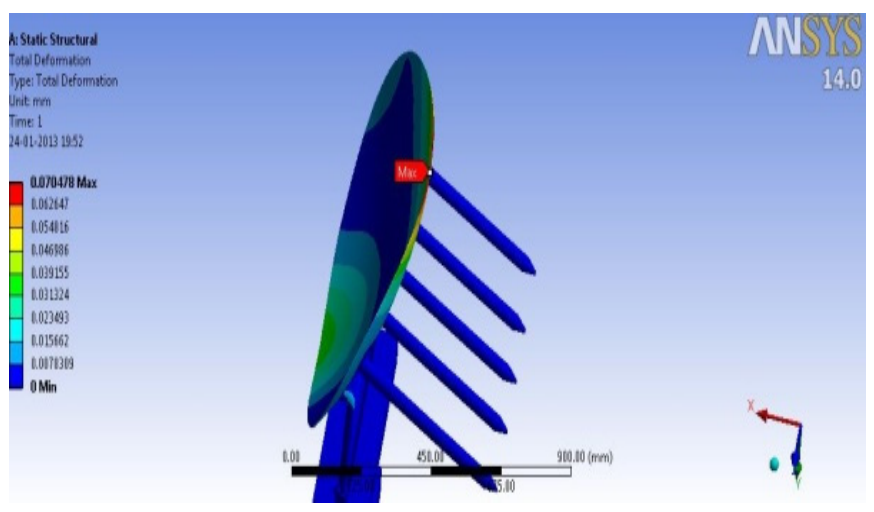

Fig. 10. Deformation Analysis

Table I shows the numerical results obtained by calculating the sample drag forces for a series of wake impact velocities and the pressure values were obtained at the plate surface, cylindrical rod and the nosecone end of the cylindrical fins. The results were found satisfactory.

TABLE I: SAMPLE PRESSURE AND FORCE CALCULATIONS

\begin{tabular}{|c|c|c|c|c|c|c|}
\hline 10 & Skin Oragforce & Inoveced drag force & a dishmajor zxisis value & resultent pressure on dish & net pressure on the plate & netpressure at tone end \\
\hline 1 & 28.8 & 19.1808 & 0.15 & 34,60264569 & 11,747132 & 9397,705598 \\
\hline 2 & 36 & 23.976 & 0.15 & 43.25330711 & 14,683915 & 11747,132 \\
\hline 3 & 43.2 & 28.7712 & 0.15 & 51,90396853 & 17,620698 & 14096.5584 \\
\hline 4 & 50.4 & 33.5664 & 0.15 & 60.55462995 & 20.55748099 & 16445.9848 \\
\hline 5 & 57.6 & 38.3616 & 0.15 & 69.20529138 & 23.494262699 & 18795.4112 \\
\hline 6 & 64.8 & 43.1568 & 0.15 & 77,8559528 & 26.43104699 & 21144.83759 \\
\hline 7 & 72 & 47.952 & 0.15 & 86.50661422 & 29.36782999 & 23494.26399 \\
\hline 8 & 79.2 & 52,7472 & 0.15 & 95.15727564 & 32.30461299 & 25843.69039 \\
\hline 9 & 86.4 & 57.5424 & 0.15 & 103.8079371 & 35.24139599 & 28193.11679 \\
\hline 10 & 108 & 71,928 & 0.15 & 129.7599213 & 44.05174499 & 35241.39599 \\
\hline
\end{tabular}

\section{CONCLUSION}

It is expected that using the above proposal it is possible to generate electricity and power up airport necessities. It will also be possible to power runway lighting. Besides that it will definitely help airports to maintain their power budget. It's a complete pollution free, safe and green technique using 
smart materials (piezoelectric) and Van de graaff principle (electrostatics) coupled to an ultra capacitor set up, for continual extraction. Surely the cost reduction as well as other benefits will be on a small scale but if implied with other emerging energy harnessing solutions will give great results. The numerical and analytical tests carried out so far have shown positive outcomes and in the era of energy crises the introduction of such smarter runways will lead to a boom in the aviation sector and also make the idea of sustainability more realistic. The initial investment is the only constraint in the introduction of such runways, although in the longer run the idea will prove to be fruitful.

\section{ACKNOWLEDGMENT}

Mohit Agarwal would foremost like to greet a vote of thanks to the second other Jafar Ali for his invaluable cooperation. The paper is the results of months of efforts. The support of the institution was immense in the completion of the research.

\section{REFERENCES}

[1] Wind Engineering Retrospect and Prospect papers of ninth International Conference, 1995 vol. 1, Theme- 2 Extreme Winds, Study of extreme wind estimation procedures. ISBN 81-224-0714-5.

[2] James F. Shackelford and Mandanapalli K. Murlidhara, Introduction to Material Science for Engineers.; Pearson Education Inc- United States 2005, ch 15 ,pp. 507.

[3] G. S. Upadhyaya and A. Upadhyaya, Material Science and Engineering; Viva Group 2006, ch. 4, article 4.2 .5 piezoelectricity pp. 137.

[4] Charrier Jacques "Le générateur de Van de Graaff," Faculté des Sciences de Nantes.

[5] Allen Mottershead, Electronic Devices and Circuits An Introduction. Eastern Economy Edition 1993, ch. 4, article 4.1-4.6.
[6] John D. Anderson, Jr. Computational Fluid Dynamics the Basics with Applications; Tata Mcgraw Hill edition 2012, ch. 5, article 5.7, pp. 193. [7] J. J. Thomsen, Vibrations and Stability Advanced Theory, Analysis and Tools; New York: Springer- Verlag 2007, appendix D, pp. 376-378.

8] CADD Centre Driving Digital Design, Ansys Reference Guide; CADD Centre Training Services Private Ltd August 2012, ch. 11, article 11.1, project 1, pp.274-291.

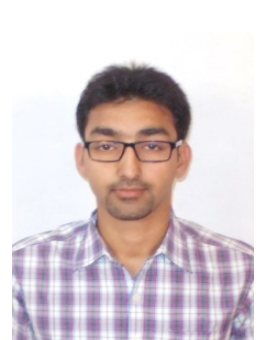

Mohit Agarwal was born on 24th April, 1992 in India, and is a citizen of India. Mohit Agarwal has completed his secondary and higher secondary education from CBSE board. $\mathrm{He}$ is a third year student of Mechanical Engineering at SRM University, located in Chennai, India. He was a participant of CANSAT competition, held in Texas, USA in the month of June 2012 . He is also a part of the 'university rover challenge team' which would be representing the college in this international competition in Colorado. Currently he is also the part of the thermal subsystem of SRMSAT-2(NANO Satellite) and responsible for the thermal control system. He is also an active member of SAE and AIAA and has participated in various technical and curricular activities at the college level.

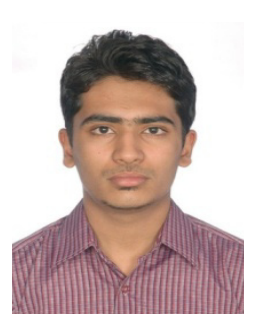

Jafar Ali Habshee was born in Udaipur, India on 19thSeptember 1992.He completed his schooling from Central Academy, Udaipur and passed his high school with $80.4 \%$. Currently he is pursuing his Bachelor in technology in aerospace engineering from SRM University Chennai Tamil Nadu. The modeling and analysis simulation of this project is done carefully under his command. He has also undergone two week training at Hindustan Aeronautical Limited in Engine division. He has participated and organized various events in his college. His main areas of interest are mainly flight dynamics and propulsion. 